\title{
Reversible Hearing Impairment Due to Inferior Colliculi Compression by a Pineal Glial Cyst
}

\author{
Yoshifumi MizOBUCHI, ${ }^{1}$ Aki SHIMADA, ${ }^{2}$ Kohei NAKAJIMA, ${ }^{1}$ \\ Hiroshi KAGUSA, ${ }^{1}$ and Yasushi TAKAGI ${ }^{1}$ \\ ${ }^{1}$ Department of Neurosurgery, Graduate School of Biomedical Sciences, \\ Tokushima University, Tokushima, Tokushima, Japan \\ ${ }^{2}$ Department of Otolaryngology, Graduate School of Biomedical Sciences, \\ Tokushima University, Tokushima, Tokushima, Japan
}

\begin{abstract}
Pineal glial cysts associated with bilateral hearing impairment are very rare. Here, we present the case of a 13-year-old boy with a pineal cyst, which caused severe bilateral hearing impairment persisting from 6 years of age. When the patient was 6 years old, the bilateral hearing acuity was about $40 \mathrm{~dB}$ on audiometry. Upon admission to our otolaryngology department, his audiogram revealed a bilateral worsening of the hearing acuity $(80 \mathrm{~dB})$. Magnetic resonance imaging (MRI) revealed an abnormal pineal cyst with tectal compression from the left with hardly normal bilateral brainstem auditory evoked potentials (BAEPs). We obtained informed consent for exploratory surgery and employed the right occipital transtentorial approach for pineal cyst removal. Based on histological examination, we diagnosed a glial cyst of the pineal gland. At 12 months postoperatively, the patient's hearing improved, showing a bilateral hearing acuity of 40 $\mathrm{dB}$ on audiometry. Since the auditory pathway has both crossed and uncrossed fibers at the upper pons and midbrain level, compression at the lateral lemniscus or inferior colliculus level can cause bilateral hearing impairment. In the present case, there was a possible slow pineal cyst growth that eventually compressed the upper pons to the midbrain, lateral lemniscuses, or inferior colliculi from the left side, this eventually led to bilateral hearing impairment. These findings indicate that surgery can improve hearing acuity in patients with a pineal cyst associated with progressive hearing impairment.
\end{abstract}

Keywords: glial cyst, hearing impairment, pineal cyst, inferior colliculi

\section{Introduction}

Non-neoplastic pineal cysts are relatively common incidental findings in healthy individuals. The prevalence of pineal cysts ranges from 1.1 to $4.3 \%$ in the adult population and $1.9 \%$ among children. ${ }^{1,2)}$ Such cysts are typically small without clinical implications with rare symptoms, including headache, diplopia, and treatment requirement. ${ }^{3)}$ In contrast, symptomatic large glial pineal cysts requiring surgical intervention without ventriculomegaly or Parinaud's syndrome are rare. ${ }^{4,5)}$ Moreover, pineal glial cysts associated with bilateral hearing

Received April 16, 2020; Accepted May 25, 2020

Copyright $\subseteq 2021$ by The Japan Neurosurgical Society This work is licensed under a Creative Commons AttributionNonCommercial-NoDerivatives International License. impairment are very rare. ${ }^{6)}$ Here, we present the case of a 13-year-old boy with a pineal cyst, which caused severe bilateral hearing impairment, without hydrocephalus or Parinaud`s syndrome, and improved postoperatively. Moreover, we discuss the possible mechanisms underlying hearing impairment, as well as the characteristics and evolution of this condition.

\section{Case Report}

A 13-year-old boy presented with hearing impairment that had persisted from 6 years of age. At the age of 6 years, the patient had bilateral hearing acuity of about $40 \mathrm{~dB}$ on audiometry. There was a gradual decrease in his hearing acuity and his academic performance worsened sharply after the age of 10 years due to progressive hearing impairment. At this point, he was admitted to the otolaryngology department in another hospital for 

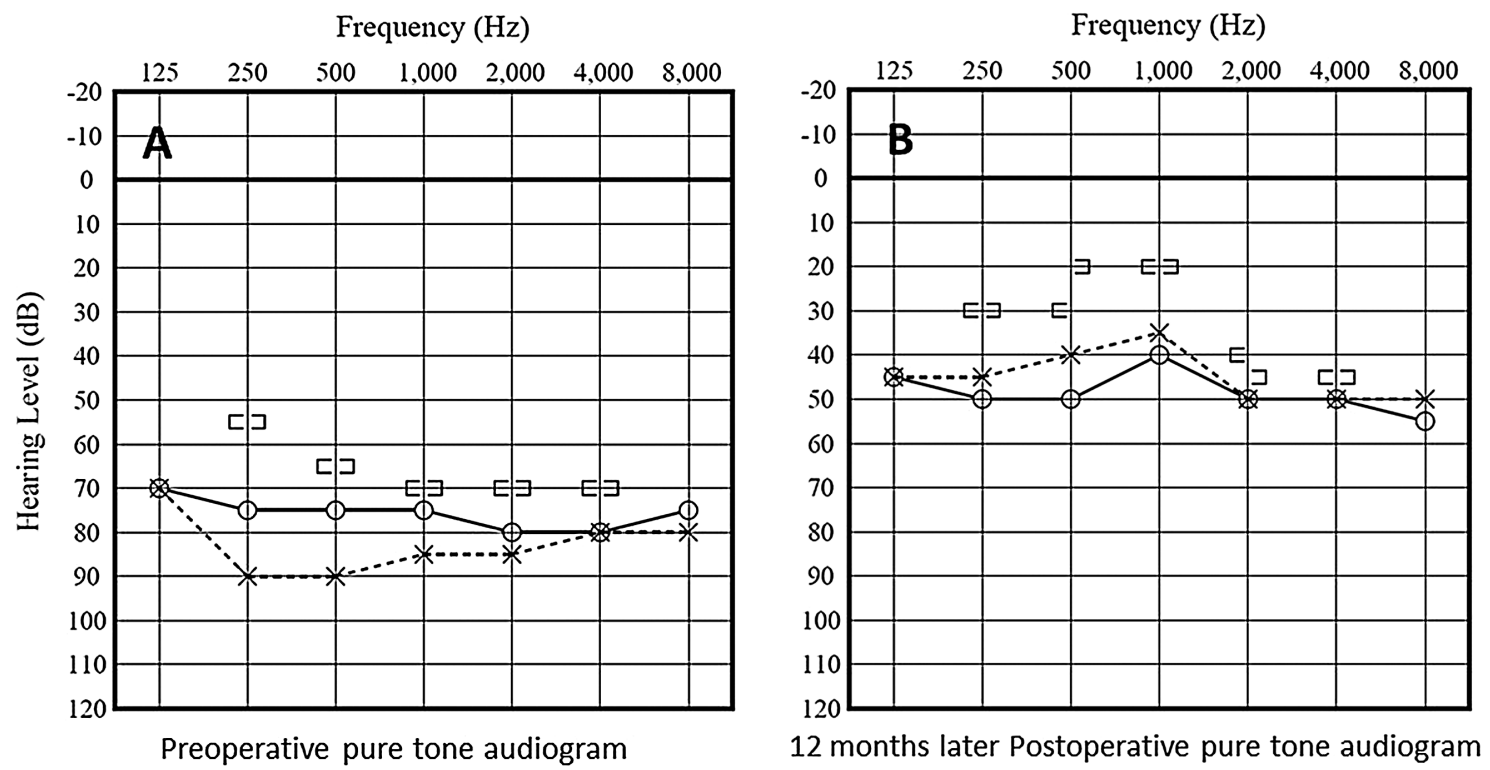

Fig. 1 Left: Preoperative pure tone audiogram showing severe bilateral hearing disturbance. Right: Postoperative pure tone audiogram obtained at the 12-month follow-up showing improved bilateral hearing acuity.



Pre op

C

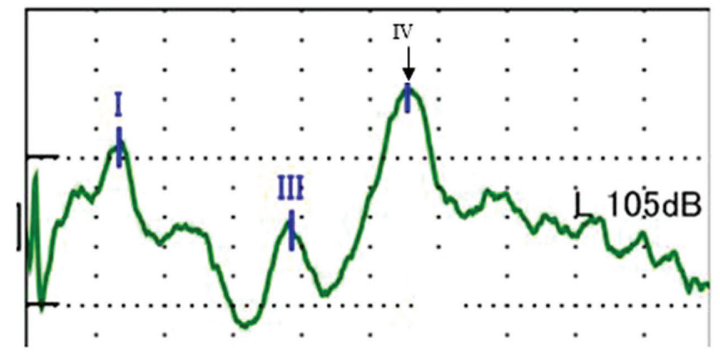

Post op

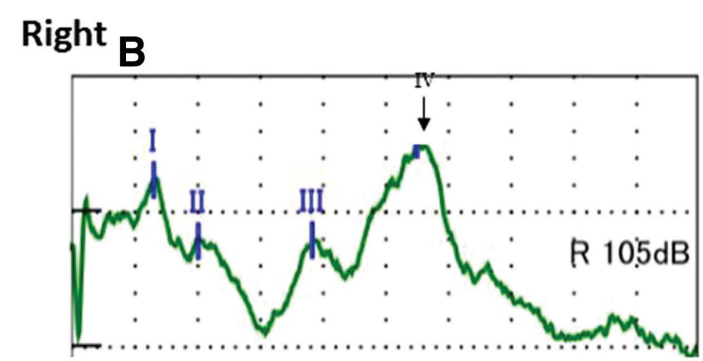

Pre op

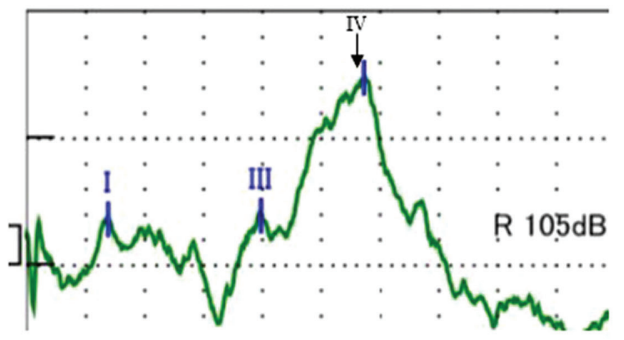

Post op

Fig. 2 Preoperative and postoperative left and right BAEPs revealing absence of wave $\mathrm{V}$ between 5 and $6 \mathrm{~m} / \mathrm{sec}$ (A-D). BAEPs: brainstem auditory evoked potentials.

progressive hearing impairment where he received conservative treatment. However, the gradual hearing deterioration remained until he required a hearing aid. On admission to the otolaryngology department of our hospital, his audiogram revealed bilateral hearing acuity worsening (80 dB) (Fig. 1, left panel) compared to the previous observation, 3 months before (60 dB). Computed tomography (CT) revealed no deformity in the middle and inner ear with vestibular aqueduct expansion in the temporal bone. Regarding the bilateral brainstem auditory evoked potentials (BAEPs), there was absence of wave $\mathrm{V}$, and this wave was hardly normal (Figs. 2A and 2B). Magnetic resonance imaging (MRI) revealed a pineal 

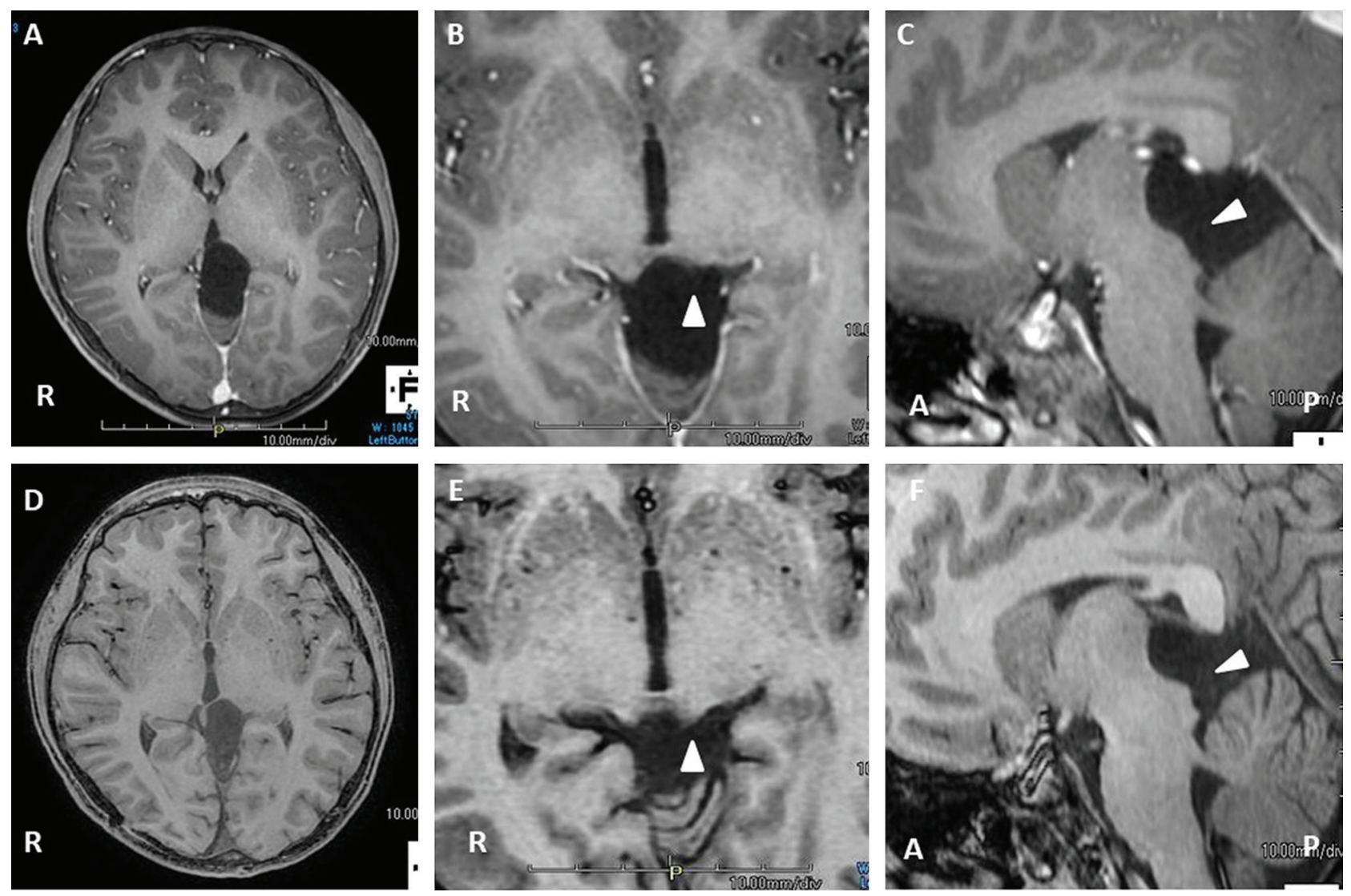

Fig. 3 Preoperative gadolinium-enhanced MRI revealing a pineal cyst $(2.5 \mathrm{~cm} \times 3.6 \mathrm{~cm} \times 3.8 \mathrm{~cm}) \mathrm{without}$ an enhanced wall (A) that compresses the brainstem and cerebellar surface, specifically the tectal plate (arrowhead) (B, C). Postoperative MRI T1-weighted images revealing relief of the compression of the brainstem and cerebellar surface (D, E, and F). MRI: magnetic resonance imaging.

cyst $(2.5 \mathrm{~cm} \times 3.6 \mathrm{~cm} \times 3.8 \mathrm{~cm})$ compressing the brainstem from the left side and cerebellar surface (Figs. 3A-3C). Subsequently, the patient was referred to the Department of Neurosurgery. Neurological examination revealed no papilledema and no Parinaud's syndrome. Moreover, there was no elevation in the levels of tumor markers, including serum human chorionic gonadotropin and alphafetoprotein. Pressure on the brainstem's dorsal hearing pathway from the cystic lesion was considered as the cause of the progressive hearing impairment. We obtained informed consent for exploratory surgery and employed the right occipital transtentorial approach for pineal cyst removal. Figure 4 presents the intraoperative views. The gray-colored cyst wall was smooth and slightly thick (Fig. 4A). We fenestrated the cyst wall, which led to the release of clear fluid, and removed the posterior wall of the pineal cyst (Fig. 4B). The anterior wall of the pineal cyst was thin, and a part of the posterior wall was fenestrated (Figs. 4C and 4D). A histological examination led to the diagnosis of a pineal glial cyst (Fig. 5). There was no clear improvement of the hearing ability immediately after the operation, but hearing ability gradually improved after 3 months. At 12 months postoperatively, there was no marked improvement in the bilateral BAEPs (Figs. 2C and 2D); however, an improvement of the patient's hearing was noted, showing a bilateral hearing acuity of $40 \mathrm{~dB}$ on audiometry (Fig. 1, right panel). Postoperative MRI revealed a relief of the brainstem's compression, especially in the tectal plate and cerebellar surface (Figs. 3D-3F).

\section{Discussion}

Pineal cysts are a common finding during routine autopsy and MRI, with an incidence of $21-40 \%$ and $1.4-4.3 \%$, respectively. The peak prevalence is observed in the age group of 19-30 years, significantly decreasing with advancing age among adults. Younger age and female sex are independent factors for increased pineal cyst prevalence in adults. The pineal cyst incidence on MRI among younger patients 

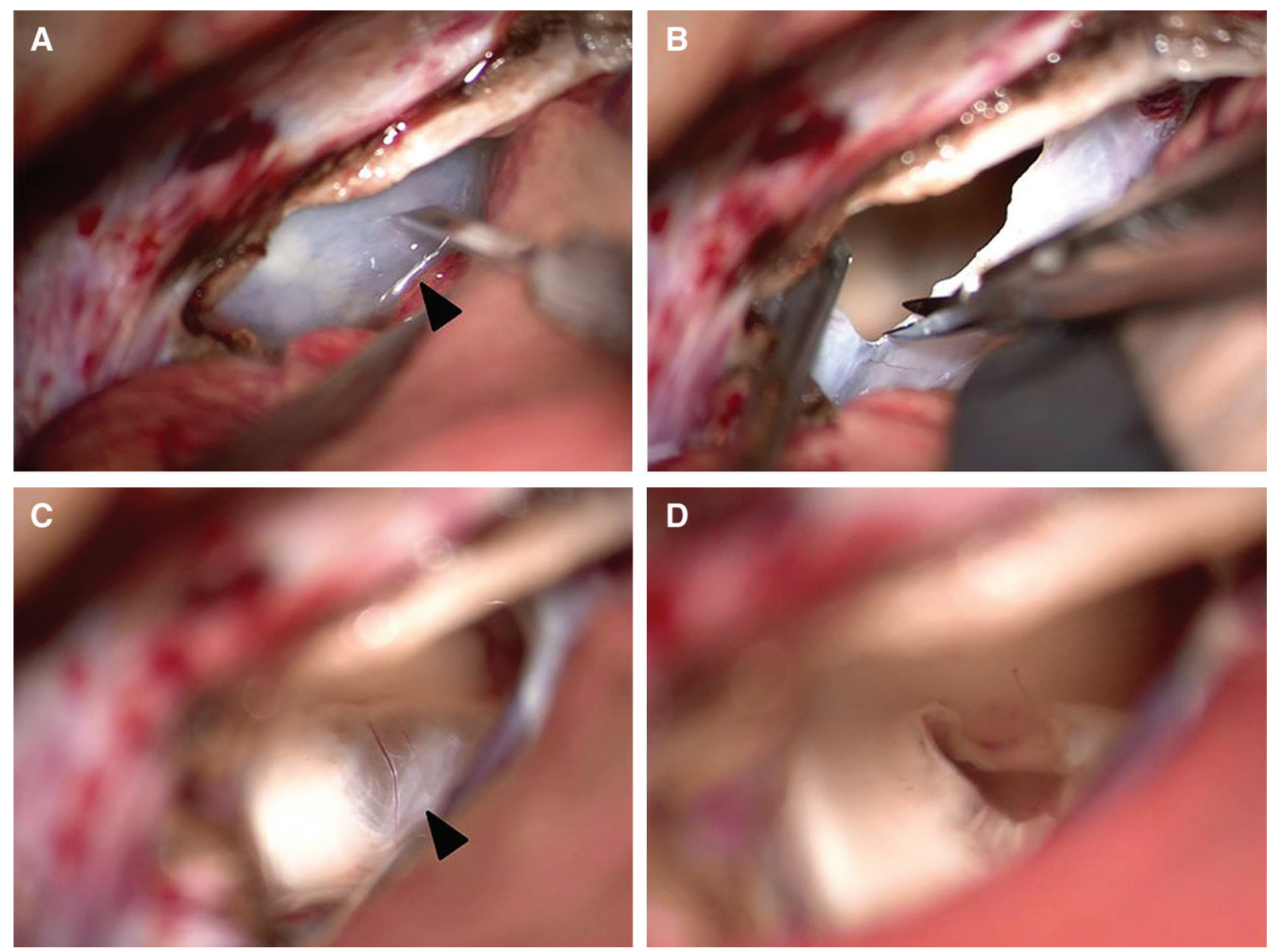

Fig. 4 Intraoperative photograph showing the gray-colored cyst wall, which was smooth and slightly thick (arrowhead) (A). The posterior wall of the pineal cyst was dissected using micro scissors (B). The anterior wall of pineal cyst was thin (arrowhead) (C) and a part of the wall was fenestrated (D).

is $1.9 \% .^{1,2,5)}$ Compared with cysts among older patients, those in younger patients are more likely to change or grow but are likely to remain asymptomatic. ${ }^{1)}$ Symptomatic pineal cysts are rare and are usually larger than $15 \mathrm{~mm}$ in diameter. $\left.{ }^{7}\right)$ Al-wolou et al. suggested that symptoms without obstructive hydrocephalus or clear signs of tectal compression should not be attributed to pineal cysts and patients should be conservatively managed. ${ }^{1)}$ Contrastingly, previous studies have suggested that obstructive hydrocephalus and Parinaud's syndrome are not required to render pineal cysts symptomatic. ${ }^{8,9)}$ Our patient had a pineal cyst larger than $30 \mathrm{~mm}$ in diameter and presented with severe progressing bilateral hearing impairment without obstructive hydrocephalus and Parinaud's syndrome. Moreover, there was no clear wave $\mathrm{V}$ in bilateral BAEPs, and it was hardly normal. BAEPs can reveal wave $\mathrm{V}$ loss or reduction in the case of midbrain or upper pons lesions. ${ }^{10)}$ However, even under normal conditions, roughly $15-20 \%$ of all cases have the wave
IV-V complex, ${ }^{11)}$ which indicates that abnormalities of BAEPs do not always imply the presence of a hearing deficit ${ }^{12,13)}$ and diagnosis of the cause of hearing impairment should not be solely based on BAEPs. In our case, there were no clear abnormal lesions in the middle or inner ear, and the patient exhibited progressive bilateral hearing loss. Moreover, MRI revealed a pineal cyst applying pressure to the midbrain and upper pons, and there was no clear wave $\mathrm{V}$ on the BAEPs. Therefore, we concluded that the progressive bilateral hearing impairment was caused by the pineal cyst.

Candidates for treatment modalities for pineal cysts are microsurgical approach, shunt surgery, and endoscopic approach. In this case, the endoscopic approach could not be used because there was no obstructive hydrocephalus caused by the pineal cyst. Moreover, it has been reported in the literature that cyst removal provides more symptom relief than shunt surgery; ${ }^{14)}$ therefore, we selected an occipital transtentorial approach for microsurgical 



Fig. 5 Photomicrograph of the cyst. Histopathology (Hematoxylin \& Eosin) (A) and immunohistochemical stains confirmed the diagnosis as a glial cyst of the pineal body. In immunohistochemical staining, the lining cells of the cyst are positive for glial fibrillar acidic protein (B) and negative for synaptophysin (C) (A, B, C: $\times 20$ ).

cyst removal. Hearing ability improved gradually after 3 months, and there was an improvement of the patient's hearing on audiometry at 12 months postoperatively. In pediatric cases, reactivity to audiometry may improve with growth, but this patient was 13 years old on admission to our institution; therefore, there was little influence of reactive improvement accompanied with growth.

Common pineal tumor symptoms include headache, papilledema, and Parinaud's syndrome, which are caused by hydrocephalus and superior colliculus compression. ${ }^{11}$ However, in our case, only bilateral hearing impairment gradually worsened. Hearing impairment is more common in pineal region meningioma than in other pineal tumors. ${ }^{15}$ The gradual growth of meningiomas might cause compression of the inferior colliculus and medial lemniscus, which causes hearing impairment without progression to hydrocephalus or Parinaud's syndrome. Although there have been a few reported cases of meningiomas in the pineal region with hearing impairment, ${ }^{6)}$ no case of bilateral hearing impairment caused by a pineal glial cyst has been reported. Pineal glial cysts are known to grow as slowly as meningiomas, unlike the rapidly growing malignant tumors, such as germ cell tumors. ${ }^{6,15)}$ In preoperative MRI, not only the inferior colliculi but also superior colliculi were compressed by the pineal glial cyst, but symptoms such as double vision or Parinaud's syndrome were not recognized. It is possible that sensory nerve fibers, such as those of the auditory pathway, were more susceptible to gradual compression than motor nerve fibers, such as those of the oculomotor nuclei; therefore, the superior and inferior colliculi were gradually compressed by the pineal region, causing only hearing impairment without Parinaud`s syndrome. In our case, there could have been slow pineal glial cyst growth, which eventually compressed the upper pons and midbrain and led to bilateral hearing impairment without Parinaud's syndrome. The auditory pathway contains both crossed and uncrossed fibers at the upper pons and midbrain level. Therefore, unilateral compression at the inferior colliculus level could cause bilateral hearing impairment. ${ }^{10)}$ In our case, MRI showed a large pineal cyst that compressed the upper pons and midbrain from the left side, which suggested compression of the lateral lemnisci and inferior colliculi and led to bilateral hearing impairment. 
Although there is no clear standard timing about the operation, if the pineal cyst is considered to be the cause of the hearing impairment, operation for pineal cyst should be performed before the condition becomes a hard of hearing impairment.

The mechanisms underlying glial cyst formation and growth remain unclear. There have been several hypotheses regarding the growth of preexisting pineal cysts, including hormonal changes and hemorrhage following pineal apoplexy. ${ }^{16,17)}$ Kang et al. demonstrated the communication between cysts and the third ventricle, with to-and-from cerebrospinal fluid movement, playing major roles in preexisting cyst growth. ${ }^{7}$ However, this hypothesis is inconsistent with our case, as we did not observe such communication.

Generally, pineal cysts are usually incidentally detected and remain asymptomatic without changes in size upon follow-up imaging assessments. However, for patients with pineal cysts associated with progressive hearing impairment, as in the present case, surgery could improve hearing acuity.

\section{Conflicts of Interest Disclosure}

The authors declare that they have no competing interests.

\section{References}

1) Al-Holou WN, Garton HJ, Muraszko KM, Ibrahim M, Maher CO: Prevalence of pineal cysts in children and young adults. Clinical article. J Neurosurg Pediatr 4: 230-236, 2009

2) Al-Holou WN, Maher CO, Muraszko KM, Garton HJL: The natural history of pineal cysts in children and young adults. J Neurosurgery Pediatr 5: 162-166, 2010

3) Taraszewska A, Matyja E, Koszewski W, Zaczyński A, Bardadin K, Czernicki Z: Asymptomatic and symptomatic glial cysts of the pineal gland. Folia Neuropathol 46: 186-195, 2008

4) El Damaty A, Fleck S, Matthes M, Baldauf J, Schroeder HWS: Pineal cyst without hydrocephalus: clinical presentation and postoperative clinical course after infratentorial supracerebellar resection. World Neurosurg 129: e530-e537, 2019

5) Al-Holou WN, Terman SW, Kilburg C, et al.: Prevalence and natural history of pineal cysts in adults. J Neurosurg 115: 1106-1114, 2011

6) Haque M, Ohata K, Tsuyuguchi N, Sakamoto S, Hara M: A case of pineal region meningioma without dural attachment, presented with bilateral hearing impairment. Acta Neurochir (Wien) 144: 209-211; discussion 211, 2002

7) Kang HS, Kim DG, Han DH: Large glial cyst of the pineal gland: a possible growth mechanism. Case report. J Neurosurg 88: 138-140, 1998

8) Kalani MY, Wilson DA, Koechlin NO, et al.: Pineal cyst resection in the absence of ventriculomegaly or Parinaud's syndrome: clinical outcomes and implications for patient selection. J Neurosurg 123: 352-356, 2015

9) Koziarski A, Podgórski A, Zieliński GM: Surgical treatment of pineal cysts in non-hydrocephalic and neurologically intact patients: selection of surgical candidates and clinical outcome. Br J Neurosurg 33: 37-42, 2019

10) Celesia GG: Hearing disorders in brainstem lesions. Handb Clin Neurol 129: 509-536, 2015

11) Chiappa KH, Gladstone KJ, Young RR: Brain stem auditory evoked responses: studies of waveform variations in 50 normal human subjects. Arch Neurol 36: 81-87, 1979

12) Hu CJ, Chan KY, Lin TJ, Hsiao SH, Chang YM, Sung SM: Traumatic brainstem deafness with normal brainstem auditory evoked potentials. Neurology 48: 1448-1451, 1997

13) Vitte E, Tankéré F, Bernat I, Zouaoui A, Lamas G, Soudant J: Midbrain deafness with normal brainstem auditory evoked potentials. Neurology 58: 970-973, 2002

14) Eide PK, Ringstad G: Results of surgery in symptomatic non-hydrocephalic pineal cysts: role of magnetic resonance imaging biomarkers indicative of central venous hypertension. Acta Neurochir (Wien) 159: 349-361, 2017

15) Gaspar N, Verschuur A, Mercier G, Couanet D, SainteRose C, Brugières L: Reversible hearing loss associated with a malignant pineal germ cell tumor. Case report. J Neurosurg 99: 587-590, 2003

16) Fain JS, Tomlinson FH, Scheithauer BW, et al.: Symptomatic glial cysts of the pineal gland. J Neurosurg 80: 454-460, 1994

17) Berhouma M, Ni H, Delabar V, et al.: Update on the management of pineal cysts: case series and a review of the literature. Neurochirurgie 61: 201-207, 2015

Corresponding author: Yoshifumi Mizobuchi, MD, PhD Department of Neurosurgery, Graduate School of Biomedical Sciences, Tokushima University, 3-18-15 Kuramotocho, Tokushima, Tokushima 770-8503, Japan.

e-mail: mizo@yj8.so-net.ne.jp 